\title{
Planificación presupuestaria basada en actividades (ABB) y su incidencia en la rentabilidad de la industria de confecciones
}

\section{Activity-based budget planning (ABB) and its impact on the profitability of the clothing industry}

\author{
Marjorie Elizabeth Tamayo Guzmán \\ mtamayog@psg.ucacue.edu.ec \\ Universidad Católica de Cuenca, Cuenca \\ Ecuador \\ https://orcid.org/0000-0002-7691-8681 \\ Cecilia Ivonne Narváez Zurita \\ inarvaez@ucacue.edu.ec \\ Universidad Católica de Cuenca, Cuenca \\ Ecuador \\ https://orcid.org/0000-0002-7437-9880 \\ Juan Carlos Erazo Álvarez \\ jcerazo@ucacue.edu.ec \\ Universidad Católica de Cuenca, Cuenca \\ Ecuador \\ https://orcid.org/0000-0001-6480-2270
}

Recibido: 8 de septiembre de 2019

Aprobado: 23 de septiembre de 2019

\section{RESUMEN}

El presupuesto es un instrumento de planificación que integra y coordina todas las áreas, actividades y departamentos de una organización, en este sentido, el presente artículo consideró como unidad de análisis de esta investigación a la industria de confecciones "Deportes Martínez" de la ciudad de Santa Rosa, provincia de El Oro, ya que en la misma se evidencia problemas de rentabilidad puesto que sus costos de producción son elevados impidiéndole competir adecuadamente en el mercado respecto al precio de venta. En virtud de lo expuesto, se plantea como objetivo diseñar un modelo de planificación presupuestaria basada actividades ABB que permita al área de producción controlar el presupuesto de inversión. Para ello, la investigación se desarrolló en un diseño no experimental bajo un enfoque mixto, en la primera fase mediante el uso de 


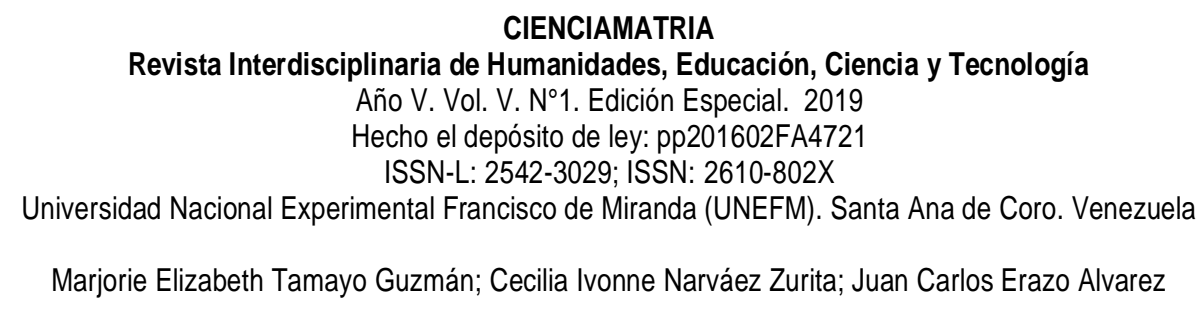

técnicas cualitativas se sustentó el marco teórico y se evaluó la situación actual de la unidad de análisis, en la segunda fase a partir del uso de técnicas cuantitativas se evaluó los mecanismos de presupuestación que aplica la empresa. Finalmente se propone la estructura del modelo de presupuestos ABB para la producción de camisetas sublimadas.

Descriptores: Presupuestos ABB; Actividades; Planificación; Presupuesto; Rentabilidad.

\begin{abstract}
The budget is a planning instrument that integrates and coordinates all the areas, activities and departments of an organization, in this sense, this article considered as a unit of analysis of this research the garment industry "Martínez Sport" of the city of Santa Rosa, province of El Oro, since it shows profitability problems since its production costs are high preventing it from adequately competing in the market with respect to the sale price. By virtue of the above, the objective is to design a budget planning model based on ABB activities that allows the production area to control the investment budget. For this, the research was carried out in a non-experimental design under a mixed approach, in the first phase through the use of qualitative techniques the theoretical framework was supported and the current situation of the unit of analysis was evaluated, in the second phase from the use of quantitative techniques, the budgeting mechanisms applied by the company were evaluated. Finally, the structure of the ABB budget model for the production of sublimated shirts is proposed.
\end{abstract}

Descriptors: Activity-Based Budgeting; Activities; Planning; Budget, Profitability.

\title{
INTRODUCCIÓN
}

En los últimos años Ecuador experimentó la contracción del consumo interno que provocó la disminución de demanda de vestimenta, pasando de un cuarto a un octavo puesto de preferencia de compra, además, dos años de inflación negativa y el descenso de la compra pública marcaron la disminución de las ventas de la industria de textiles y confecciones.

Al respecto la Asociación de Industrias Textiles del Ecuador (AITE), señala que, para el año 2018 las pequeñas y grandes empresas empezaron a mejorar sus condiciones en el mercado a partir de un buen manejo de su patrimonio, endeudamiento y apalancamiento 


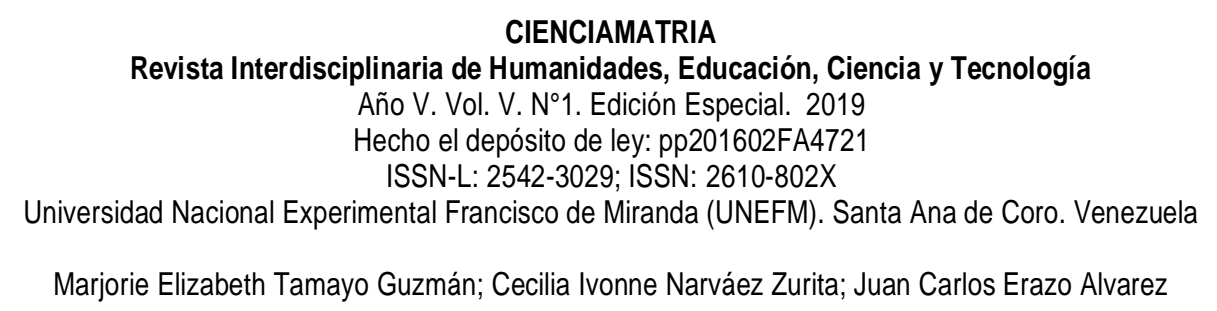

de sus negocios, sin embargo, aún se evidencia factores complejos como el nivel de ventas, las cuentas por cobrar a clientes, la planificación de la producción y las estimaciones presupuestarias.

En atención a lo expuesto, se puede alegar que; la Industria Deportes Martínez, ubicada en la ciudad de Santa Rosa, dedicada exclusivamente a la fabricación de ropa deportiva sublimada, en los últimos años ha tenido que hacerle frente a los excesivos gastos en materia prima, y a la falta de integración entre los procesos de planeación estratégica y de presupuestación, lo que ha impedido prever la cantidad de recursos necesarios para el proceso de producción.

Sobre la base de las consideraciones anteriores, se puede acotar que la unidad de análisis según diagnóstico realizado presenta actualmente dificultades en el manejo administrativo relacionado con las ventas, el control de la mano de obra y la adquisición de materiales, para la confección de camisetas sublimadas, como consecuencia de la deficiente planificación y la carencia de un plan de acción que ha conllevado a la pérdida de posicionamiento en el mercado.

Ante estos sucesos, el presente artículo tiene como objetivo diseñar un modelo de planificación presupuestaria basada en actividades (ABB) que permite el mejoramiento de la rentabilidad de la Industria "Deportes Martínez" de la ciudad de Santa Rosa, optimizando recursos y garantizando la correcta comercialización de las prendas deportivas.

\section{DESARROLLO}

El presupuesto es un instrumento de planificación que, de una forma categórica, realiza la integración y coordinación de las áreas, actividades, departamentos y responsables de una organización (Muñiz, 2009), así también, el presupuesto expresa en términos monetarios los ingresos y gastos en la búsqueda de un fin que es planificar entradas y salidas de dinero. Por su parte, Rincón (2011) señala que, el presupuesto es entregable 


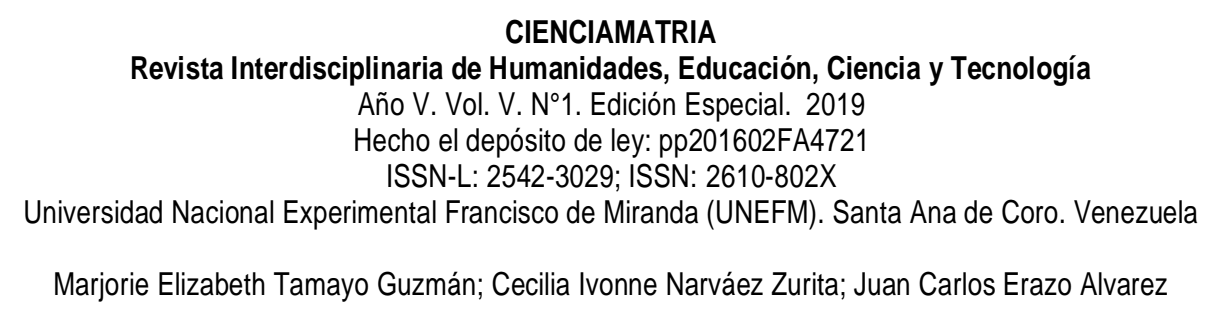

como resultado a indicadores financieros sobre la cantidad y el costo de los recursos necesarios para desarrollar un producto o alguna actividad, buscando prever el futuro. Sin duda alguna, un presupuesto es importante porque permite trazar planes, metas y objetivos, siguiendo líneas de discusión, aprobación, ejecución, control y de evaluación de procesos o actividades en una línea de tiempo, obteniendo datos más certeros y así poder ejecutar un control (Jazmín y Pacheco, 2015). Un presupuesto es una mirada al panorama del futuro (Toro, 2010). Por ello, un presupuesto permite que las industrias tengan un alto nivel de productividad, gestión de los recursos y el alcance de los objetivos; al identificar aquellas áreas fuertes y débiles de un todo (Cubillan y Colina, 2013), encontrándose la planificación presupuestaria enmarcada por cinco principios (ver tabla 1).

Tabla 1

Principios de la planificación presupuestaria Principio Detalle

De previsión Relativo a la importancia del estudio anticipado de las cosas y de las posibilidades de lograr metas propuestas.

De planeación Referente al camino trazado para lograr los objetivos deseado, alineado a la exactitud, contabilización, transigencia, unidad, seguridad, cooperación, oportunidad.

De organización Concerniente a las actividades a fin de alcanzar las metas.

De dirección Pertinente a la manera de dirigir las acciones de orden y comunicación hacia el cumplimiento de los objetivos esperados.

De control Relativo a las comparaciones entre lo planificado y alcanzado.

En numerosas pequeñas y medianas industrias el presupuesto constituye la planificación anual y se convierte, además, en el instrumento de comunicación de los objetivos industriales a todos los niveles de la organización. Una industria correctamente encaminada comúnmente sigue este curso en la preparación del presupuesto: (1) planifica las subunidades que participan en el desarrollo de la Industria, (2) determina un marco de referencia de expectativas específicas, (3) controlan las desviaciones del plan 
por medio de acciones correctivas, y (4) planifica continuamente a partir de la retroalimentación y variaciones de un caso a otro (Toro, 2010).

La planificación presupuestaria tiene como objetivo básico trasladar a presupuestos y metas por departamento, áreas o centros de costos de la industria, las estimaciones generales de la planificación estratégica (ibídem).

\section{PRESUPUESTO BASADO EN ACTIVIDADES: ABC VS ABB}

Según Urquía y Lorain (2008), para preparar un presupuesto basado en actividades es necesario reconocer las causas de los costos y las características de los productos y servicios; ya que estás consideran que varíen los costos de los procesos, a partir de la búsqueda de los inductores de actividad, los cuales permiten planificar y medir los recursos necesarios, realizando el análisis de las características del producto, en la línea de la planificación estratégica. Así mismo, este autor menciona que el proceso ABB invierte las relaciones causales de un modelo ABC (Ver figura 1).

\section{Figura 1}

Modelo ABC vs. ABB

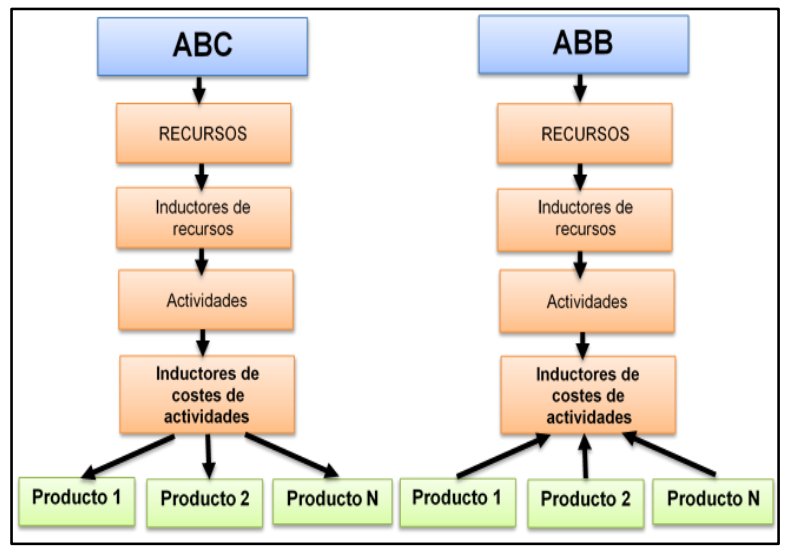

La adaptación de la filosofía $A B C$ y $A B M$ al proceso presupuestario se denomina presupuesto en base a actividades (Activity based Budgesting-ABB). Con el ABB el presupuesto se centra en las actividades desarrolladas en la industria, siendo una herramienta financiera por su aporte a una eficiente gestión de los recursos, permitiendo 


\section{CIENCIAMATRIA \\ Revista Interdisciplinaria de Humanidades, Educación, Ciencia y Tecnología \\ Año V. Vol. V. №1. Edición Especial. 2019 \\ Hecho el depósito de ley: pp201602FA4721 \\ ISSN-L: 2542-3029; ISSN: 2610-802X \\ Universidad Nacional Experimental Francisco de Miranda (UNEFM). Santa Ana de Coro. Venezuela \\ Marjorie Elizabeth Tamayo Guzmán; Cecilia Ivonne Narváez Zurita; Juan Carlos Erazo Alvarez}

a la gerencia tener un mayor control sobre estos ya que posee como antecedente las demandas pronosticadas para las actividades desarrolladas. En sí, se refiere al proceso de planificación y presupuesto entorno a una lógica enfocada a los procesos que convierte de forma más concreta los ejes estratégicos desarrollados por la industria (Urquía y Lorain, 2008).

Kaplan y Anderson (2008), afirman que la elaboración de presupuestos basados en ABB permite a las Industrias prever los cambios en las demandas de recursos a partir de las eficiencias de procesos previstos y a partir de los cambios en el volumen y la combinación de las transacciones. La elaboración de presupuestos basados en actividades elimina buena parte de las negociaciones y regateos asociados al proceso tradicional de elaboración de presupuestos detallados.

EI ABB inicia con la proyección de los volúmenes de los diversos objetos de costos a ser fabricados en una línea determinada del tiempo, para en seguida calcular el valor económico que necesitan las actividades para saciar estos volúmenes mediante el uso de los generadores de costo y de los artilugios de filtración de costos de las actividades ligadas a un elemento de costo (Toro, 2010). Sobre la base de estas ideas, Cuervo, Osorio, y Duque (2013) señalan que existen procesos que son necesarios para obtener un presupuesto en base de actividades, los mismos que se detallan en la tabla 2.

\section{Tabla 2}

Fases para elaborar un presupuesto en base a las actividades

\begin{tabular}{cl}
\hline Fase & \multicolumn{1}{c}{ Detalle } \\
\hline Primera & $\begin{array}{l}\text { Identificar las actividades diversas que realiza una industria para } \\
\text { vender un producto o servicio, uniendolas por funciones y } \\
\text { procesos, desarrollando así una ruta de actividades. }\end{array}$ \\
\hline Segunda & $\begin{array}{l}\text { Determinar los costos requeridos para llevar a cabo cada } \\
\text { actividad en cada proceso en cada área con base a los insumos } \\
\text { que requiere cada obra. }\end{array}$ \\
\hline Tercera & $\begin{array}{l}\text { Establecer la demanda con base en el presupuesto, las } \\
\text { actividades unitarias, así como la utilización del costo de cada } \\
\text { actividad, nivel productivo y la posibiliadad de crear productos y } \\
\text { servicios nuevos. }\end{array}$ \\
\hline Cuarta & Calcular los costos necesarios en todo el proceso de producción. \\
\hline
\end{tabular}


Quinta Detallar los presupuestos como costos de cada unidad de la industria

Fuente: (Cuervo, Osorio, y Duque, 2013).

El ABB se enfoca en el costo estimado de las actividades demandas para producir y vender la producción (Pietrzak, 2013). Por su parte, autores como Salas y Soldevila (2015), presentan otros procesos para poder elaborar un presupuesto en base a las actividades (ver tabla 3 ).

Tabla 3

Procesos para elaborar un presupuesto en base actividades

Acción

Detalle

Estimar Las unidades a vender y producir de acuerdo a los objetivos.

Considerar Los inductores de costos de actividades y el número de los mismos para las actividades fundamentales.
Estimar El número de inductores de las actividades auxiliares o secundarias
Determinar Los costos de actividades principales y auxiliares o secundarias en relación a los recursos necesarios para el desarrollo de las actividades.
Clasificar En caso que la industria utilice centros de costos, se deberá clasificar las actividades.

Presupuestar Los diferentes centros de costos más presupuestos de costos directos representan el total del presupuesto de la Industria

Fuente: (Salas y Soldevila, 2015).

Mora (2009) asevera una clasificación de las actividades, en 2 categorías, cada una con su sub clasificación respectiva: (1) por jerarquía; subclasificada en: macro actividades y micro actividades, y (2) por nivel; subclasificada en: nivel unitario, de lote, de línea y de apoyo. La clasificación por jerarquía busca el orden en cada etapa de procesamiento de la información, para una mejor comprensión en la tabla 4 se detalla los niveles jerárquicos. 


\section{CIENCIAMATRIA}

Revista Interdisciplinaria de Humanidades, Educación, Ciencia y Tecnología

Año V. Vol. V. №1. Edición Especial. 2019

Hecho el depósito de ley: pp201602FA4721

ISSN-L: 2542-3029; ISSN: 2610-802X

Universidad Nacional Experimental Francisco de Miranda (UNEFM). Santa Ana de Coro. Venezuela

Marjorie Elizabeth Tamayo Guzmán; Cecilia Ivonne Narváez Zurita; Juan Carlos Erazo Alvarez

Tabla 4

Sub clasificación del nivel jerárquico

\begin{tabular}{cl}
\hline Macro actividades & \multicolumn{1}{c}{ Micro actividades } \\
\hline Son las acciones que cimentan los procesos & $\begin{array}{l}\text { Operaciones: identifican a los objetivos claves de } \\
\text { los procesos. }\end{array}$
\end{tabular}

Éstas disminuyen en su nivel jerárquico en base a su funcionalidad y necesidad.
Tareas: son actividades más bajas del nivel jerárquico. Únicamente se reemplazan cuando se detecte que su accionar permite reconocer los elementos propios necesarios a utilizar.

Mientras que, la clasificación de las actividades por nivel vincula intrínsecamente las actividades con el objetivo de cada una de ellas, esta clasificación cobra especial importancia por cuanto permite tener una visión más amplia al normalizar el costo habitual de la actividad, de manera relativa a la cantidad de factores necesarios con el objetivo productivo de la misma, dada en relación del número de inductores que esta acción podría lograr en un periodo de tiempo (Ver figura 2).

\section{Figura 2}

Actividades clasificadas por nivel

\begin{tabular}{|c|c|}
\hline & $\begin{array}{l}\text { Nivel unitario: costos y acciones están direccionadas al logro } \\
\text { de una unidad de objetivo productivo sea esta manufacturada o } \\
\text { de servicio. }\end{array}$ \\
\hline & $\begin{array}{l}\text { Nivel de lote: tienen como objetivo un lote. No necesariamente } \\
\text { tiene que ser un lote de venta, sino que son acciones cuyos } \\
\text { costos de sus recursos consumibles están asociados al logro de } \\
\text { un lote específico }\end{array}$ \\
\hline & $\begin{array}{l}\text { Nivel de línea: estas acciones asocian sus costos y su } \\
\text { desarrollo al mantenimiento de las líneas específicas } \\
\text { generadoras de objetivos productivos o de servicios. Sus } \\
\text { inductores estarán asociados con las líneas de producción de } \\
\text { generación de servicios disponibles. }\end{array}$ \\
\hline & $\begin{array}{l}\text { Nivel de apoyo o soporte: estas acciones soportan alos } \\
\text { procesos productivos. Son actividades esenciales, sin las cuales } \\
\text { las empresas no podrían funcionar, pero no se encuentran } \\
\text { dentro de la secuencia de obtención de los objetivos productivos. }\end{array}$ \\
\hline
\end{tabular}




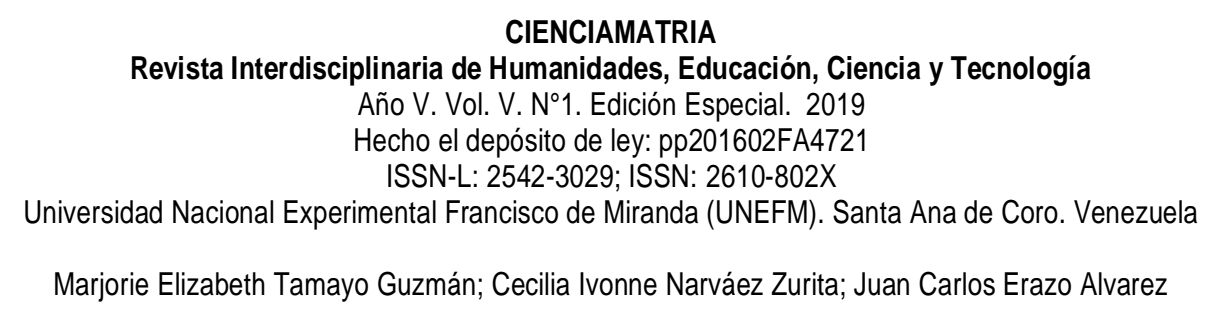

Las actividades se definen como la secuencia de tareas para lograr un objetivo. En este escenario, Rodríguez (2008) clasifica las actividades directas e indirectas o también conocidas como de apoyo. Las actividades directas son aquellas que benefician directamente a la producción; mientras que las actividades de apoyo se identifican como el conjunto de tareas relacionadas, que no son parte del proceso productivo, por ejemplo: actividades realizadas por la parte administrativa.

Por otra parte, Möller (2011) afirma que las actividades se clasifican de acuerdo a lo que se quiere analizar: a) en relación de la actuación con el producto, b) conforme al entorno de acción, c) en función de su habilidad para añadir valor agregado al producto, d) según la periodicidad de realización, e) según la función de la unidad en la que se realizan, f) por su peso en los factores de éxito.

Así mismo, Mora (2009) contempla una clasificación de los inductores en secuenciales y de apoyo. Los inductores secuenciales se originan en las actividades de producción, los mismos que se determinan en el control de los procesos, y normalmente a través de la identificación de sus causas, mientras que, los inductores de apoyo se establecen al identificar las actividades de apoyo de los procesos productivos. Suelen tener un alto grado de dificultad para ser identificados ya que se debe analizar su vinculación con los procesos productivos.

\section{CONTROL DE PRESUPUESTO BASADO EN ACTIVIDADES}

Es impetuoso manejar un control presupuestario efectivo, y ante ello, se deben seguir las siguiente recomendaciones: (1) comprobar si la finalidad del presupuesto se ha cumplido, (2) evidenciar y estimar en qué etapa no se han cumplido los objetivos presupuestarios, (3) examinar si los objetivos planteados con anterioridad son adaptables al contexto de la organización y (4) calificar cómo han reaccionado los responsables ante las situaciones encontradas (Muñiz, 2012).

En base a lo expuesto anteriormente, se hace indispensable el conocimiento de aspectos teóricos de los costos ABC. Dentro de este marco, Arellano, Quispe, Ayaviri, y Escobar 
(2017) afirman que el sistema de costos $A B C$ determina los costos de las actividades basado en el uso de los recursos y establece costos a los objetos de costos, de acuerdo al consumo de cada una de las actividades; por ello, es importante considerar la identificación de recursos, actividades, asignación de inductores y centro de costos.

También es necesario mencionar que el ABM es una herramienta para la toma de decisiones, que permite a la administración de una industria mejorar mediante la optimización y reducción de los costos, desarrollando ventajas competitivas y alcanzando metas de rentabilidad. El ABM, no solamente se enfoca en el logro de la excelencia industrial, sino también, en el sostenimiento de la misma, exigiendo una mejora continua de todas las actividades de la industria, tanto de las áreas productivas como la administrativa. Es así que, el $A B C$ evalúa la viabilidad, mientras que el $A B M$ garantiza la viabilidad (Cuervo, Osorio, y Duque, 2013).

Los costos $A B C$ se relacionan con el $A B M$ al fijar los costos por actividades y decidir sobre aspectos tales como: la reducción de costos, la dirección o planificación estratégica, la merma de desperdicios, el desarrollo de acciones competitivas, el manejo de forma adecuada de la estrategia de precios, y la generación de información que permita elegir si desarrollar o comprar un bien. Por tanto, el ABM se enfoca en la gestión y excelencia de las Industrias mientras que, el $A B C$ alcanza sus objetivos a través de dos subcomponentes adjuntos dentro del ABM: operativo y estratégico (Mejía y Salazar, 2015).

\section{METODOLOGÍA}

La investigación se abordó desde un diseño no experimental bajo una metodología de campo, asumiendo procesos del paradigma cuali-cualitativo, el mismo que siguió la lógica de la observación, medición y tratamiento estadístico de los datos recolectados en un solo momento del tiempo, por tanto su alcance fue explicativo - secuencial.

Además, se empleó el método histórico - lógico, al fundamentar en la línea del tiempo las variables de investigación a través de fuentes literarias en el contexto del valor de la 
información, se utilizó el método inductivo - deductivo al iniciar con teorías generales sobre la planificación, los presupuestos, la planificación presupuestaria basada en actividades llegando a sus fases y clasificación de actividades. Finalmente, se aplicó el método analítico - sintético en la descomposición de la planificación presupuestaria y su incidencia en la rentabilidad

Para el caso de estudio propuesto, la población estuvo conformada por la totalidad de trabajadores de la industria Deportes Martínez, que asciende a 10 personas.

\section{RESULTADOS}

En correspondencia con el objetivo de la investigación, a continuación se presenta de manera sintetizada el análisis e interpretación de los datos a partir de la aplicación de encuestas y entrevistas:

- La empresa carece de un adecuado tratamiento contable sobre sus operaciones, lo que ha ocasionado que la unidad de análisis presente inconvenientes al momento de tomar decisiones por no poseer conocimiento oportuno sobre sus resultados económicos obtenidos.

- El cuerpo directorio desconoce sobre los costos de producción de la industria, por ende no tiene un adecuado funcionamiento administrativo

- Los procedimientos administrativos que emplea la industria no permiten planificar, coordinar y controlar la adquisición de materia prima, o insumos indirectos de producción necesarios para sus operaciones.

- No existe una planificación presupuestaria en la industria, que permita controlar oportunamente las operaciones administrativas relacionadas con los costos, gastos, ventas y utilidades.

- No es adecuada la forma como la industria administra los requerimientos de personal, ni el número de horas necesarias para cada orden de producción. 
Ante los resultados expuestos se determina la necesidad de estructurar un modelo de presupuestos basados en actividades que permita el mejoramiento de la rentabilidad de la industria "Deportes Martínez". El modelo contiene 5 componentes que permiten la interrelación sistemica entre la planificación y la presupuestación (ver figura 3).

Figura 3

Esquema de la propuesta para la planificación presupuestaria basada en actividades ABB.

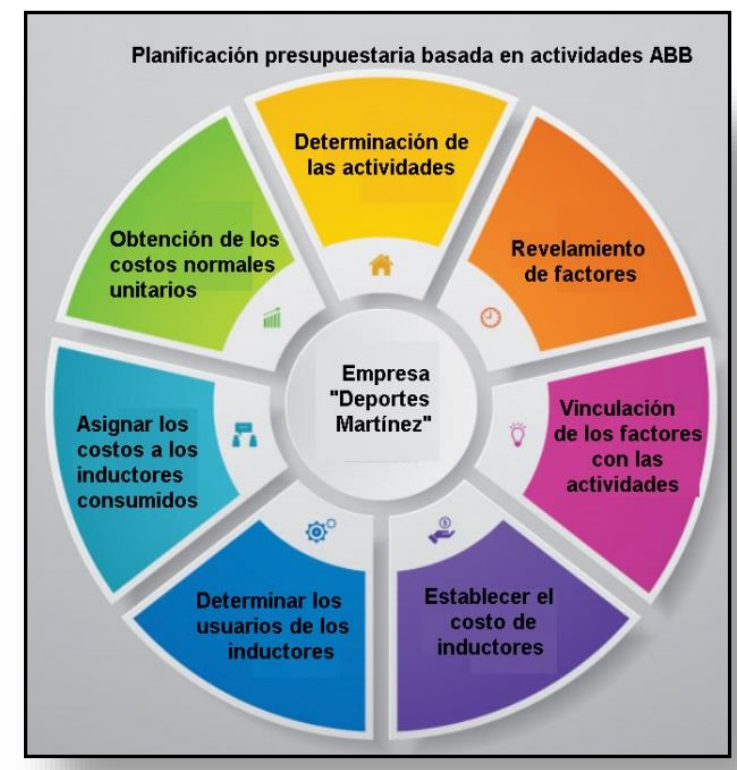

- Determinación de las actividades: es un punto clave para realizar la planificación presupuestaria, identificar las actividades necesarias dentro del proceso productivo de la industria, y probar si se complementan y así facilitar el entendimiento de este método y la importancia de la información revelada.

- Identificación de factores: las actividades determinadas, requieren factores para sistematizar su capacidad, la cual es tipificada en número de inductores recabados en un tiempo definido. De tal modo, se procede al descubrimiento del coste de los factores necesarios a utilizar.

- Vinculación de los factores con las actividades: en el costo por actividad permanece la información de la suma de factores necesarios para ejecutar la actividad y lograr la cantidad de inductores presupuestados. 


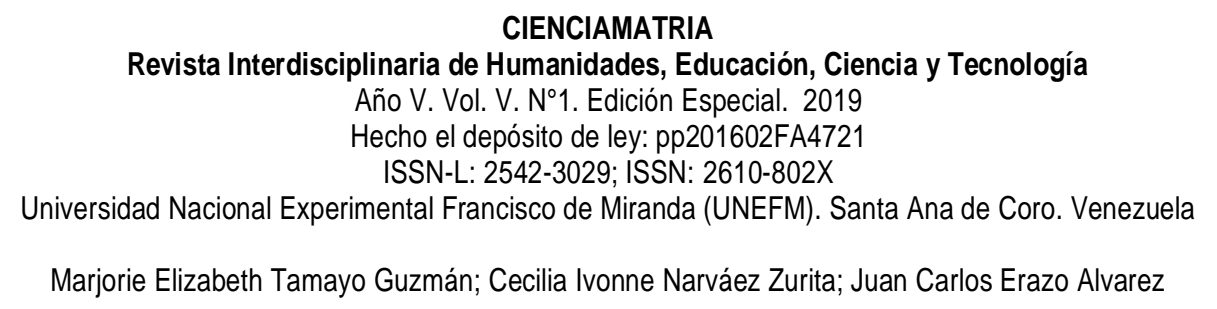

- Fijación del costo de inductores: el costo del inductor se normaliza al obtener el coste total de cada actividad y la cantidad de inductores que cada una puede obtener.

- Establecimiento de los tipos de inductores: el siguiente movimiento es determinar cuáles son los inductores, esto con la finalidad de conocer la regulación del número a emplear.

- Asignación de costos a los inductores: permite la incorporación de los costos de las actividades al costo de los productos.

- Obtención de los costos unitarios: una vez desarrolladas todas estas instrucciones, se obtendrán los costos totales y unitarios por cada objetivo.

Para la obtención de los datos de los costos a través de este método involucra la realización de un presupuesto por cada una de las actividades determinadas en la matriz de acumulación de costos. Esta técnica es la consecuencia de la utilización por parte del proceso de los inductores regulares que cada actividad determine, y ante ello es necesario habilitar un sistema de información para brindar informes combinados para usos distintos, Ortega Polanco (2017).

En la figura 4. Se detallan las actividades determinadas en el proceso de producción de la industria "Deportes Martínez".

\section{Figura 4}

Determinación de actividades

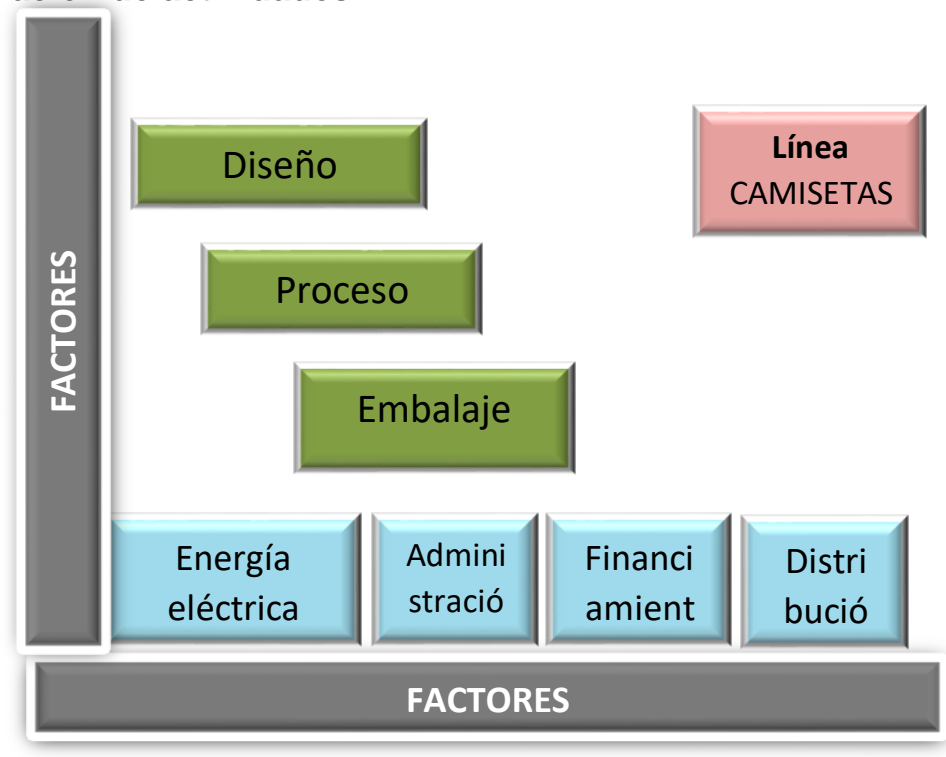




\section{CIENCIAMATRIA}

Revista Interdisciplinaria de Humanidades, Educación, Ciencia y Tecnología

Año V. Vol. V. №1. Edición Especial. 2019

Hecho el depósito de ley: pp201602FA4721

ISSN-L: 2542-3029; ISSN: 2610-802X

Universidad Nacional Experimental Francisco de Miranda (UNEFM). Santa Ana de Coro. Venezuela

Marjorie Elizabeth Tamayo Guzmán; Cecilia Ivonne Narváez Zurita; Juan Carlos Erazo Alvarez

Enseguida, se determina el posible consumo de factores con sus respectivos componentes físicos y monetarios (ver tabla 5).

Tabla 5

Consumo de factores

\begin{tabular}{|c|c|c|c|c|c|c|c|c|c|c|}
\hline Código & Detalle & & Vida útil & & Valor & $\begin{array}{l}\text { Component } \\
\text { e Físico }\end{array}$ & $\begin{array}{r}\text { Com } \\
\text { mo }\end{array}$ & $\begin{array}{l}\text { ponente } \\
\text { petario }\end{array}$ & & Total \\
\hline 1 & Diseñador & & & & & 1 salario & $\$$ & 800.00 & $\$$ & 800.00 \\
\hline 2 & Administrador & & & & & 1 Salario & $\begin{array}{l}\$ \\
1000\end{array}$ & .00 & $\begin{array}{l}\$ \\
10\end{array}$ & 0.00 \\
\hline 3 & Operarios & & & & & 5 salarios & $\$$ & 500.00 & $\$$ & 2500.00 \\
\hline 4 & Honorarios & & & & & $\begin{array}{c}1 \\
\text { honorario }\end{array}$ & $\$$ & 600.00 & $\$$ & 600.00 \\
\hline 5 & Insumos & & & & & 1 Kit & $\$$ & 300.00 & $\$$ & 300.00 \\
\hline 6 & $\begin{array}{l}\text { Suministros } \\
\text { oficina }\end{array}$ & de & & & & $1 \mathrm{Kit}$ & $\$$ & 100.00 & $\$$ & 100.00 \\
\hline 7 & $\begin{array}{l}\text { Equipo } \\
\text { computación }\end{array}$ & de & 3 años & $\$$ & 1500.00 & $1 \mathrm{PC}$ & $\begin{array}{l}\$ \\
41.6\end{array}$ & & & $\begin{array}{c}\$ \\
41.67\end{array}$ \\
\hline 8 & $\begin{array}{l}\text { Muebles } \\
\text { oficina }\end{array}$ & de & 10 años & $\$$ & 1800.00 & 1 unidad & & $\begin{array}{l}\$ \\
5.00\end{array}$ & & $\begin{array}{c}\$ \\
15.00\end{array}$ \\
\hline 9 & $\begin{array}{l}\text { Maquinaria } \\
\text { equipo }\end{array}$ & $y$ & 10 años & $\$$ & 40000.00 & 1 unidad & $\$$ & 333.33 & $\$$ & 333.33 \\
\hline 10 & $\begin{array}{l}\text { Consumo } \\
\text { energía }\end{array}$ & de & & & & $6000 \mathrm{~kW} / \mathrm{h}$ & $\begin{array}{l}\$ \\
0.05\end{array}$ & & $\$$ & 00.05 \\
\hline 11 & $\begin{array}{l}\text { Capital } \\
\text { terceros }\end{array}$ & de & & & $30,000.00$ & & & mes & $\$$ & 300.00 \\
\hline 12 & $\begin{array}{l}\text { Instalaciones } \\
\text { fabrica }\end{array}$ & de & 20 años & & $70,000.00$ & $\begin{array}{c}1 \\
\text { instalación }\end{array}$ & $\$$ & 291.67 & $\$$ & 291.67 \\
\hline
\end{tabular}

Fuente: Deportes Martínez

Determinadas las actividades, y luego de analizar el consumo de los factores de acuerdo a lo establecido como meta por la industria, se procede a estructurar la base de datos de las actividades (ver tabla 6). 


\section{CIENCIAMATRIA}

Revista Interdisciplinaria de Humanidades, Educación, Ciencia y Tecnología

Año V. Vol. V. №1. Edición Especial. 2019

Hecho el depósito de ley: pp201602FA4721

ISSN-L: 2542-3029; ISSN: 2610-802X

Universidad Nacional Experimental Francisco de Miranda (UNEFM). Santa Ana de Coro. Venezuela

Marjorie Elizabeth Tamayo Guzmán; Cecilia Ivonne Narváez Zurita; Juan Carlos Erazo Alvarez

Tabla 6

Base de datos de las actividades

\begin{tabular}{|c|c|c|c|c|c|}
\hline Código & Descripción & Jerarquía & Nivel & Inductor & Capacidad \\
\hline 1 & Diseño & $\begin{array}{l}\text { Producción } \\
\text { Camiseta }\end{array}$ & Unitaria & Unidad & 1200 \\
\hline 2 & $\begin{array}{l}\text { Proceso de corte y } \\
\text { armado }\end{array}$ & $\begin{array}{l}\text { Producción } \\
\text { Camiseta }\end{array}$ & Línea & Unidad & 1200 \\
\hline 3 & Embalaje & $\begin{array}{l}\text { Producción } \\
\text { Camiseta }\end{array}$ & Unitaria & Unidad & 1200 \\
\hline 4 & Energía eléctrica & Apoyo & Apoyo & $\mathrm{kW} / \mathrm{h}$ & $6000 \mathrm{~kW} / \mathrm{h}$ \\
\hline 5 & Administración & Apoyo & Apoyo & $\begin{array}{l}\text { Horas } \\
\text { laboradas }\end{array}$ & $200 h$ \\
\hline 6 & Financiamiento & Apoyo & Apoyo & $\begin{array}{l}\text { Capital } \\
\text { asignado }\end{array}$ & $\$ 30,000.00$ \\
\hline 7 & Distribución & Apoyo & Apoyo & $\begin{array}{l}\text { Horas } \\
\text { vendedor }\end{array}$ & $200 \mathrm{~h}$ \\
\hline
\end{tabular}

Los costos de los factores $1,5,6,7,9$ y 12 han sido utilizados en el desarrollo de la actividad de diseño, generando que el coste de los recursos tanto físico como monetario represente el costo total de la actividad de diseño. Si al total, le afecta la cantidad de inductores que se obtienen mensualmente, en este caso ascendieron a 1200 camisetas, entonces, se conoció el costo de cada inductor, es decir, de cada camiseta (ver figura 5).

\section{Figura 5}

Consumo de los factores por las actividades

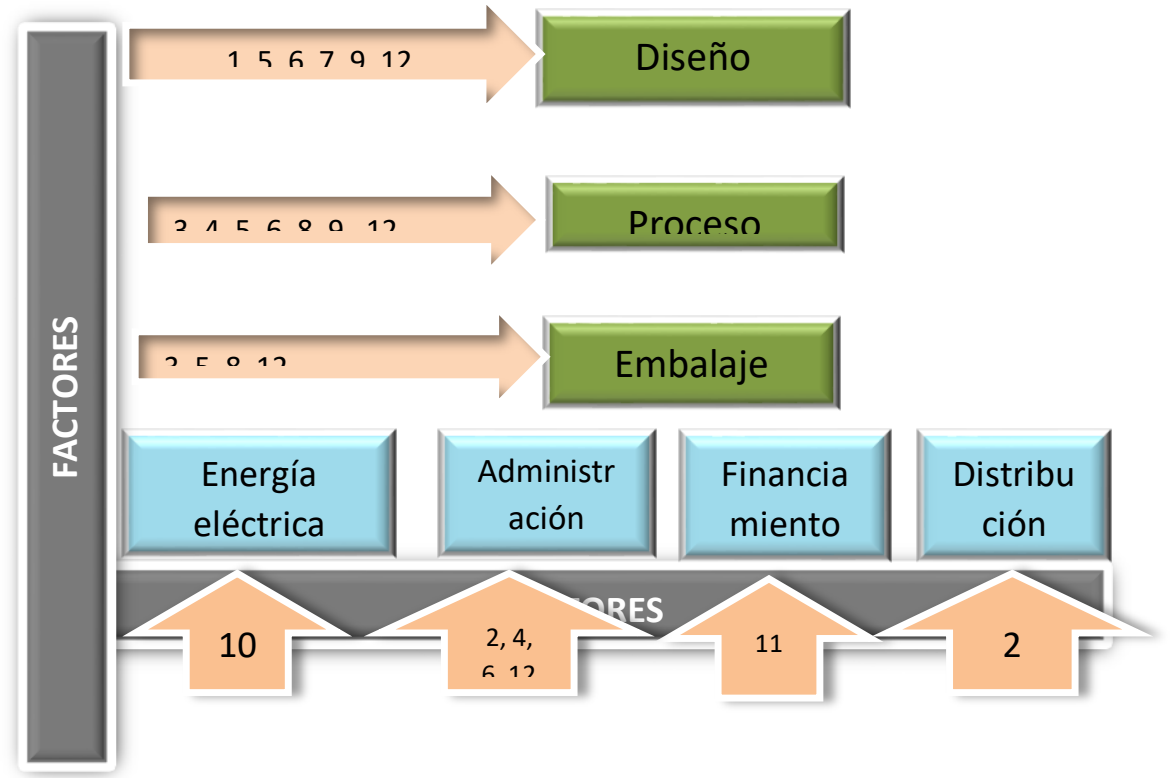


Es necesario que en la elaboración del presupuesto por actividades, se normalice cada actividad con los recursos necesarios para el funcionamiento, y por supuesto continuar el curso de la actividad generadora de costes del proceso por la utilización de los factores, Rodríguez \& Puertas (2017).

Respecto a estos consumos, se expresa el total de costos por cada actividad y el costo unitario que desprende cada inductor (ver tabla 7)

Tabla 7

Costos totales de las actividades Código Descripción

1 Diseño

2 Proceso de corte y armado Costo total Inductor

$\$ 1214.59$

$\$ 2680.42$

1200
Costo inductor

3 Embalaje $\$ 680.42$

1200

4 Suministro de energías

$\$ 300.00$

1200

5 Administración

$\$ 906.25$

6 Financiamiento

$\$ 300.00$

6000

0.5670

7 Distribución

$\$ 500.00$

$150 \mathrm{HH}$

30000

$160 \mathrm{HH}$

$\$ 0.01 \times$ cada $\$$ aplicado

Los costos identificados en cada una de las actividades fijan el punto de partida del presupuesto basado en actividades y en esta fase de la propuesta falta identificar las actividades de apoyo, con el fin de conseguir el presupuesto final de cada actividad (ver tabla 8)

Tabla 8

Actividades de apoyo

Suministro de energía

$3000 \mathrm{~kW} / \mathrm{h}$ Diseño

$3000 \mathrm{~kW} / \mathrm{h}$ Proceso de corte y armado

Administración

50 HHE Proceso de adquisición de Materia prima

50 HHE Proceso de corte y armado

50 HHE Embalaje

Distribución

160 HHE Logística para entregar el producto

HEE: Horas trabajo Administrador 
Se desarrollaron los presupuestos de todas las actividades definidas en el proceso de confección de camisetas. Comenzando con las actividades que requieren de energía eléctrica y distribución, las mismas son actividades de apoyo que influyen en el proceso productivo, mientras que las actividades de financiamiento y administración se mantienen por separado con el fin de conservar la estructura de la propuesta en las normas contables (ver tabla 9)

\section{Tabla 9}

Presupuesto de costos por actividades de apoyo

\section{Suministro de energía}

\begin{tabular}{|c|c|c|c|c|}
\hline Diseño & Impresión de Diseño & 3000 & \multicolumn{2}{|c|}{$\$ 150.00$} \\
\hline & Estampado de Diseño & $\mathrm{kW} / \mathrm{h}$ & \multirow{2}{*}{\multicolumn{2}{|c|}{$\$ 150.00$}} \\
\hline Proceso de corte y armado & $\begin{array}{l}\text { Corte de telas } \\
\text { Armado de camiseta }\end{array}$ & $\begin{array}{l}3000 \\
\mathrm{~kW} / \mathrm{h}\end{array}$ & & \\
\hline Costo por kW/h & & & $\$$ & 0.05 \\
\hline \multicolumn{5}{|c|}{ Distribución } \\
\hline Logística para distribución & \multirow{2}{*}{\multicolumn{2}{|c|}{ 160HHE }} & \multicolumn{2}{|c|}{$\$ 500.00$} \\
\hline Costo por Hora Laborada & & & & 3.125 \\
\hline
\end{tabular}

\section{Administración}

Administrador

$\begin{array}{lr}\$ & 500.00 \\ \$ & 300.00 \\ \$ & 33.33 \\ \$ & 72.92\end{array}$

Honorarios

Suministros de oficina

Instalaciones

Total presupuesto Administración

$\$ 906.25$

\section{Financiamiento}

\section{Capital de terceros}

Del mismo modo se presenta el presupuesto de las actividades principales para la elaboración de camisetas como son: actividad de diseño (ver tabla 10), actividad de corte y armado (ver tabla 11) y actividad de embalaje (ver tabla 12). 
Revista Interdisciplinaria de Humanidades, Educación, Ciencia y Tecnología

Año V. Vol. V. Nº1. Edición Especial. 2019

Hecho el depósito de ley: pp201602FA4721

ISSN-L: 2542-3029; ISSN: 2610-802X

Universidad Nacional Experimental Francisco de Miranda (UNEFM). Santa Ana de Coro. Venezuela

Marjorie Elizabeth Tamayo Guzmán; Cecilia Ivonne Narváez Zurita; Juan Carlos Erazo Alvarez

Tabla 10

Presupuesto de costo por actividades: Diseño

\begin{tabular}{lrl} 
& Diseño \\
\hline Diseñador & $\$ 800.00$ & \\
Insumos & $\$ 100.00$ & \\
Suministros de oficina & $\$ 33.33$ & \\
Computadora & $\$ 41.67$ & \\
Maquinaria y equipo & $\$ 166.67$ & \\
Instalaciones de Fábrica & $\$ 72.92$ & $\$ 1,214.59$ \\
Costo total de diseño & & \\
\hline
\end{tabular}

\section{Tabla 11}

Presupuesto de costo por actividades: Proceso de corte y armado

Proceso de corte y armado

$\begin{array}{llr}\text { Operarios } & \$ & 2,000.00 \\ \text { Horas Contador } & \$ & 300.00 \\ \text { Insumos } & \$ & 100.00 \\ \text { Suministro de administración } & \$ & 33.33 \\ \text { Muebles de oficina } & \$ & 7.50 \\ \text { Maquinaria y equipo } & \$ & 166.67 \\ \text { Energía eléctrica } & \$ & 150.00 \\ \text { Instalaciones de fabrica } & \$ & 72.92\end{array}$

Costo total de Proceso de corte y armado $\quad \$ 2,830.42$

Tabla 12

Presupuesto de costo por actividades: Proceso de embalaje

Embalaje

Operario
Insumos
Muebles de oficina
Instalaciones de fábrica
Costo total Proceso de Embalaje

$\begin{array}{rr}\$ & 500.00 \\ \$ & 100.00 \\ \$ & 7.50 \\ \$ & 72.92\end{array}$




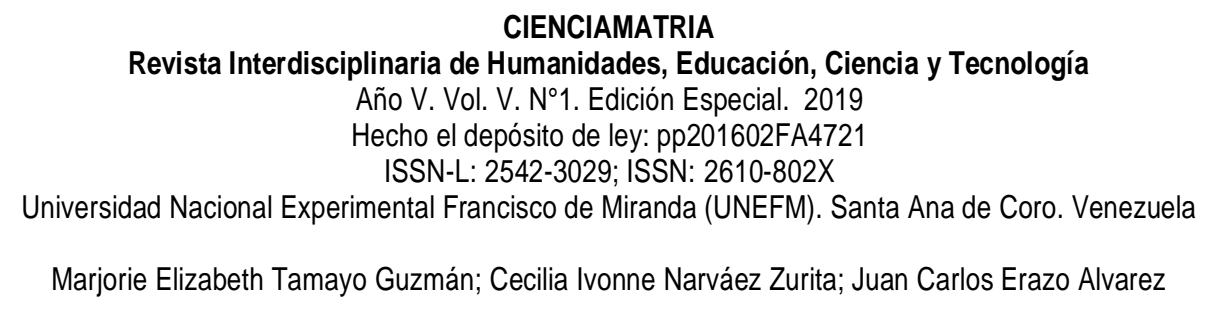

Al establecer la asignación de costos para cada actividad ya sean estas principales o de apoyo se obtiene el costo de producción total para la elaboración de 1200 camisetas, determinando el costo unitario de cada camiseta elaborada (ver tabla 13).

\section{Tabla 13}

Costo de producción de camisetas por actividades

\section{Costo Producción de Camisetas}

\begin{tabular}{lrr}
\hline Actividad de Diseño & $\$ 1,364.59$ & \\
Actividad de Corte y armado & $\$ 2,830.42$ & \\
Actividad de Embalaje & $\$ \quad 680.42$ & \\
Costo total de producción de camisetas & & $\$ 4,875.43$ \\
Costo por unidad de Camiseta & & $\$ \quad 4.06$ \\
\hline
\end{tabular}

Para la elaboración del presupuesto de ventas se toma de referencia las unidades que la industria "Deportes Martínez" estima producir en periodo de un mes (ver tabla 14).

Tabla 14

Presupuestos de ventas

Presupuesto de ventas

\begin{tabular}{llllll}
\hline Ingresos por venta de camisetas & 1200 unidades/mes & $\$$ & 7.50 & $\$$ & $9,000.00$
\end{tabular}

Ingresos totales mensuales $\quad \$ \quad 9,000.00$

Al tener como fuente de información el presupuesto de ventas en donde revela el total de ingresos por ventas y el presupuesto de cada una de las actividades para los costos, se presenta el estado de resultados proyectado por actividades (Ver tabla 15).

\section{Tabla 15}

Estado de resultados proyectado

\section{Ingresos}

Venta de camisetas (1200 uni a 7.50)

Total ingresos por ventas

Costos de Producción de camisetas

Total costos de producción por actividades
$\$ 9,000.00$

$\$ 9,000.00$

$\$ \quad 4,875.43$

$\$ 4,875.43$ 
Costos actividades de apoyo

Costos de administración

$\$ 906.25$

Costos de financiamiento

$\$ \quad 300.00$

Total costos de las actividades de apoyo

\section{DISCUSIÓN}

El presupuesto basado en actividades realizado a la Industria "Deportes Martínez", presenta las siguientes conclusiones:

- Los cambios trascendentales en la estructura de las actividades obedecen a los cambios de los procesos de corte y embalaje de las camisetas.

- Los presupuestos basados en actividades, generan un nivel alto de análisis de la información financiera aportando valor agregado a la industria, en cuanto les permite tomar decisiones eficientes gracias a identificar que costos innecesarios representan gran nivel de mala manipulación de recursos.

- La herramienta de presupuesto basado en actividades, aporta para la industria el desarrollo de estados proyectados con la finalidad de evaluar los resultados económicos principales para inducirlos como objetivos industriales

\section{REFERENCIAS CONSULTADAS}

1. Arellano, O., Quispe, G., Ayaviri, D., \& Escobar, F. (2017). Estudio de la Aplicación del Método de Costos ABC en las Mypes del Ecuador. Revista de Investigaciones Altoandinas, 19(1), 33-46. Obtenido de http://www.scielo.org.pe/scielo.php?script=sci_arttext\&pid=S231329572017000100004

2. Burbano, J. (2005). Presupuestos: enfoque de gestion, planeacion y control de recursos (Tercera edicion ed.). Colombia: MCGRAW-HILL. Recuperado el 20 de 06 de 2018, de https://catedrafinancierags.files.wordpress.com/2015/03/burbanopresupuestos-enfoque-de-gestic3b3n.pdf 


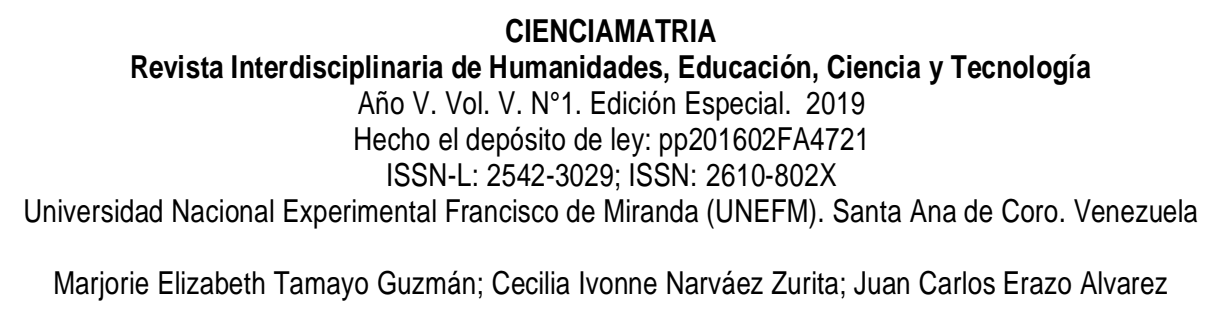

3. Cubillan, A., \& Colina, L. (2013). La planificación presupuestaria en universidades públicas. CICAG: Revista del Centro de Investigación de Ciencias Administrativas y Gerenciales, 10(1), 185-201. Obtenido de https://dialnet.unirioja.es/servlet/articulo?codigo $=5028137$

4. Cuervo, J., Osorio, J., \& Duque, M. (2013). Costeo basado en actividades ABC: Gestión basada en actividades ABM. Ecoe Ediciones. Obtenido de https://books.google.com.ec/books?id=08G4DQAAQBAJ\&dq=pasos+para+elabor ar+un+presupuesto+en+base+a+las+actividades+ABB\&source=gbs_navlinks_s

5. Moller, Y. (2011). La Contabilidad de gestión y el presupuesto basado en actividades. (U. d. Cienfuegos", Ed.) Matanza, Cuba.

6. Jazmín, D., \& Pacheco, M. (2015). Control presupuestario en La Universidad del Zulia, Venezuela. Actualidad Contable Faces, 18(31), 58-79. Obtenido de https://www.redalyc.org/pdf/257/25743363004.pdf

7. Kaplan, R., \& Anderson, S. (2008). Los presupuestos basados en actividades. Harvard Deusto Finanzas y Contabiliadad, 4-20. Recuperado el 18 de 06 de 2018, de http://www.alaracha.psdeg-psoe.org/archivos/10archivo.pdf

8. Mejía, C., \& Salazar, C. (2015). Costo de servir como variable de decisión estratégica en el diseño de estrategias de atención a canales de mercados emergentes. Estudios Gerenciales, 31(134), 50-61. doi:https://doi.org/10.1016/j.estger.2014.08.006

9. Mora, A. (2009). Diccionario de Contabilidad, Auditoría y Control de Gestión, Volumen $3 . \quad$ ECOBOOK. Obtenido de https://books.google.com.ec/books?id=ig9zAgAAQBAJ\&dq=clasificacion+de+las +actividades+por+nivel+en+las+Industrias+nivel+unitario+nivel+de+lote\&source= gbs_navlinks_s

10. Muñiz, L. (2009). Control Presupuestario. Barcelona: Profit editorial.

11. Muñiz, L. (2012). Cómo implantar y evaluar un sistema de control de gestión: Incluye cuestionarios de evaluación. Profit Editorial. Obtenido de https://books.google.com.ec/books?id=TgvJc07W0V4C\&dq=control+y+seguimien $\underline{\text { to }+ \text { de+un+presupuesto \&source }=\text { gbs navlinks } s}$

12. Ortega Polanco, V. (2017). Gestión de la imagen corporativa de organizaciones universitarias desde el enfoque del marketing emocional. CIENCIAMATRIA, 3(5), 150-171. https://doi.org/10.35381/cm.v3i5.19 


\begin{abstract}
CIENCIAMATRIA
Revista Interdisciplinaria de Humanidades, Educación, Ciencia y Tecnología

Año V. Vol. V. №1. Edición Especial. 2019

Hecho el depósito de ley: pp201602FA4721

ISSN-L: 2542-3029; ISSN: 2610-802X

Universidad Nacional Experimental Francisco de Miranda (UNEFM). Santa Ana de Coro. Venezuela

Marjorie Elizabeth Tamayo Guzmán; Cecilia Ivonne Narváez Zurita; Juan Carlos Erazo Alvarez
\end{abstract}

13. Pietrzak, Z. (2013). Traditional versus Activity-based Budgeting in Nonmanufacturing Companies. Social Sciences, 82(4), 26-37. doi:https://doi.org/10.5755/j01.ss.82.4.6604

14. Rincon Soto, C. (2011). Presupuestos Industriariales (Primera edicion ed.). Bogota, Colombia: ECO-EDICIONES.

15. Rodríguez Ceballo, L., \& Ultra Hernández, M. (2009). Enfoque de la presupuestación por las actividades en la formación de profesionales en Centro de formacon las Tunas. Obtenido de http://www.eumed.net/cursecon/ecolat/cu/2009/rcuh.htm

16. Rodríguez Rodríguez, S., Crúz, Y., \& Puertas, A. (2017). Indicadores para la comercialización de productos derivados de lotes caprinos (capra hircus), hacia una estrategia endógena de marketing en Colina - Zamora, Falcón, Venezuela.. Revista Arbitrada Interdisciplinaria Koinonía, 2(3), 198--212. Recuperado de http://fundacionkoinonia.com.ve/ojs/index.php/revistakoinonia/article/view/63/50

17. Salas, O., \& Soldevila, P. (2015). Contabilidad y gestión de costes: con ejercicios resueltos. Profit Editorial. Obtenido de https://mail.google.com/mail/u/0/?tab=rm\&ogbl\#inbox/FMfcgxwDqxPSwpfRjPnRD wmKwdttFgKk

18. Toro, F. (2010). Costos ABC y presupuestos (Primera ed.). Bogota, Colombia: Ecoe Ediciones.

19. Urquía, E., \& Lorain, M. (Noviembre de 2008). Es el fin del presupuesto? Una revision sobre las nuevas tendencias sobre presupuestación. Control de gestion(204), 84 a 97. Recuperado el 21 de 06 de 2018, de file:///C:/Users/Usuario/Desktop/MAESTRIA/BIBLIOGRAFIA/presupuesto.pdf

\title{
REFERENCES CONSULTED
}

1. Arellano, O., Quispe, G., Ayaviri, D., \& Escobar, F. (2017). Study of the Application of the ABC Cost Method in the Mypes of Ecuador. Altoandinas Investigations Magazine, $19 \quad$ (1), 33-46. $\quad$ Retrieved from http://www.scielo.org.pe/scielo.php?script=sci_arttext\&pid=S231329572017000100004 


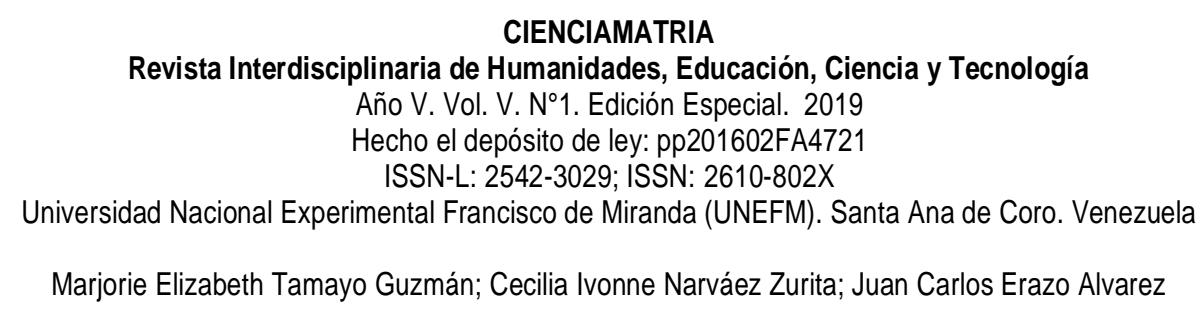

2. Burbano, J. (2005). Budgets: management, planning and resource control approach (Third edition ed.). Colombia: MCGRAW-HILL. Retrieved on June 20, 2018, from https://catedrafinancierags.files.wordpress.com/2015/03/burbanopresupuestos-enfoque-de-gestic3b3n.pdf

3. Cubillan, A., \& Colina, L. (2013). Budget planning in public universities. CICAG: Journal of the Center for Research in Administrative and Management Sciences, 10 (1), 185-201. Retrieved from https://dialnet.unirioja.es/servlet/articulo?codigo $=5028137$

4. Cuervo, J., Osorio, J., \& Duque, M. (2013). Costs based on ABC activities: Management based on ABM activities. Ecoe Editions. Retrieved from https://books.google.com.ec/books?id=08G4DQAAQBAJ\&dq=pasos+para+elabor ar+un+budget+en+base+a+las+activities+ABB\&source=gbs_navlinks_s

5. Moller, Y. (2011). Management Accounting and activity-based budget. (U. d. Cienfuegos ", Ed.) Matanza, Cuba.

6. Jasmine, D., \& Pacheco, M. (2015). Budget control at the University of Zulia, Venezuela. Accounting Faces, 18 (31), 58-79. Retrieved from https://www.redalyc.org/pdf/257/25743363004.pdf

7. Kaplan, R., \& Anderson, S. (2008). Activity-based budgets. Harvard Deusto Finance and Accounting, 4-20. Retrieved on June 18, 2018, from http://www.alaracha.psdeg-psoe.org/archivos/10archivo.pdf

8. Mejía, C., \& Salazar, C. (2015). Cost of serving as a strategic decision variable in the design of strategies for attention to emerging market channels. Management Studies, 31 (134), 50-61. doi: https: //doi.org/10.1016/j.estger.2014.08.006

9. Mora, A. (2009). Dictionary of Accounting, Audit and Management Control, Volume 3. ECOBOOK. Retrieved from https://books.google.com.ec/books?id=ig9zAgAAQBAJ\&dq=classification+of+the +activities+by+level+en+las+Industries+level+unit+level+de+lote\&source=gbs_na vlinks_s

10. Muñiz, L. (2009). Budget Control. Barcelona: Editorial Profit.

11. Muñiz, L. (2012). How to implement and evaluate a management control system: Includes evaluation questionnaires. Editorial Profit. Retrieved from https://books.google.com.ec/books?id=TgvJc07W0V4C\&dq=control+y+segment+ $\underline{\text { de}+u n+b u d g e t \& s o u r c e=g b s}$ navlinks $s$ 


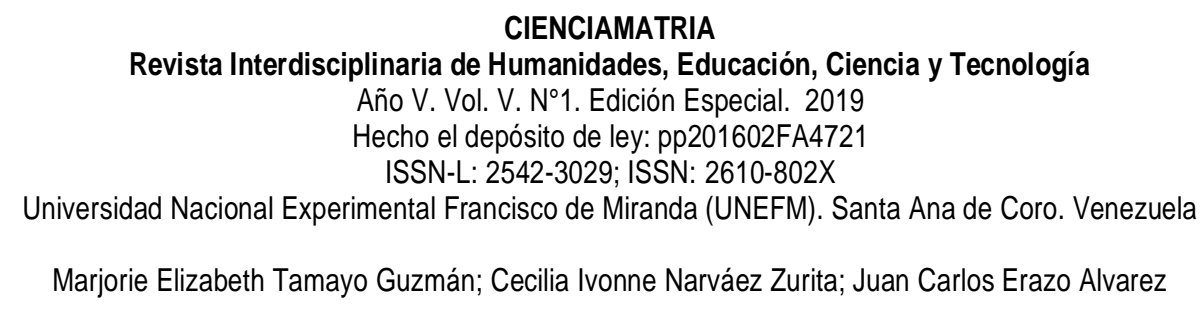

12. Ortega Polanco, V. (2017). Management of the corporate image of university organizations from the focus of emotional marketing. SCIENCE, 3 (5), 150-171. https://doi.org/10.35381/cm.v3i5.19

13. Pietrzak, Z. (2013). Traditional versus Activity-based Budgeting in Nonmanufacturing Companies. Social Sciences, 82 (4), 26-37. doi: https: //doi.org/10.5755/j01.ss.82.4.6604

14. Rincon Soto, C. (2011). Industrial Budgets (First edition ed.). Bogota, Colombia: ECO-EDITIONS.

15. Rodriguez Ceballo, L., \& Ultra Hernandez, M. (2009). Budgeting approach for activities in the training of professionals in Centro de formacon las Tunas. Retrieved from http://www.eumed.net/cursecon/ecolat/cu/2009/rcuh.htm

16. Rodríguez Rodríguez, S., Crúz, Y., \& Puertas, A. (2017). Indicadores para la comercialización de productos derivados de lotes caprinos (capra hircus), hacia una estrategia endógena de marketing en Colina - Zamora, Falcón, Venezuela.. Revista Arbitrada Interdisciplinaria Koinonía, 2(3), 198--212. Recuperado de http://fundacionkoinonia.com.ve/ojs/index.php/revistakoinonia/article/view/63/50

17. Salas, O., \& Soldevila, P. (2015). Accounting and cost management: with exercises resolved. Editorial Profit. Retrieved from https://mail.google.com/mail/u/0/?tab=rm\&ogbl\#inbox/FMfcgxwDqxPSwpfRjPnRD wmKwdttFgKk

18. Toro, F. (2010). ABC costs and budgets (First ed.). Bogota, Colombia: Ecoe Editions.

19. Urquía, E., \& Lorain, M. (November 2008). Is it the end of the budget? A review of the new trends in budgeting. Management control (204), 84 to 97. Retrieved on June 21, 2018, from file: I/I C: /Users/Usuario/Desktop/MAESTRIA/BIBLIOGRAFIA/presupuesto.pdf

(C)2019 por los autores. Este artículo es de acceso abierto y distribuido según los términos y condiciones de la licencia Creative Commons Atribución-NoComercial-Compartirlgual 4.0 Internacional (CC BY-NC-

SA 4.0) (https://creativecommons.org/licenses/by-nc-sa/4.0/). 